\title{
A PARAMETERIZATION OF THE PERIOD 3 HYPERBOLIC COMPONENTS OF THE MANDELBROT SET
}

\author{
DANTE GIARRUSSO AND YUVAL FISHER
}

(Communicated by Linda Keen)

ABSTRACT. We demonstrate that the period 3 hyperbolic components of the Mandelbrot set consist of the image of the unit disk by the maps

$$
-\frac{7}{4}-\frac{20}{9}\left[\sinh \left(\omega(z)+\frac{2 k \pi i}{3}\right)-\frac{1}{4 \sqrt{5}}\right]^{2},
$$

with

$$
\omega(z)=\frac{1}{3} \operatorname{Arcsinh}\left(\frac{88-27 z}{80 \sqrt{5}}\right),
$$

for $k=0,1,2$.

\section{INTRODUCTION}

If $f: \mathbf{C} \rightarrow \mathbf{C}$ is a holomorphic map and $z$ satisfies $f(z)=z$, then $z$ is called an attractive, repelling, or indifferent fixed point when $\left|f^{\prime}(z)\right|<1,\left|f^{\prime}(z)\right|>1$, or $\left|f^{\prime}(z)\right|=1$, respectively (see [2]). By considering the $n$-fold composition $f^{\circ n}$, the periodic points, those points satisfying $f^{\circ n}(z)=z$, can be classified in the same way. It is an amazing fact that the repelling periodic cycles outnumber the attractive cycles dramatically. In fact, an attractive periodic cycle in the complex plane will always attract a critical point; that is, if $\xi \in \mathbf{C}$ is an attractive periodic point, then it is a limit point of the sequence $\left(f^{\circ n}(x)\right)_{n}$, for some point $x \in \mathbf{C}$ satisfying $f^{\prime}(x)=0$. Thus, the quadratic family $P_{c}(z)=z^{2}+c$ can have at most one attractive periodic cycle in the plane, and this cycle will attract the critical point 0 .

When the iterates of the critical point remain bounded, the closure of all the repelling periodic points (the Julia set) will be a connected set. This is the motivation for the definition of the Mandelbrot set

$$
M=\left\{c \mid P_{c}^{o n}(0) \nrightarrow \infty \text { as } n \rightarrow \infty\right\} .
$$

Received by the editors May 19, 1994; this paper was presented on January 13, 1994 to the American Mathematical Society, at the Joint Mathematics Meeting, Special Session on Global Analysis, Cincinnati, Ohio.

1991 Mathematics Subject Classification. Primary 30C20, 30 D05.

Key words and phrases. Iterative dynamics, Mandelbrot set, hyperbolic components, factorization.

The second author was supported by DOE contract DE-FG03-90ER418.

(C)1995 American Mathematical Society 
It is a compact subset of $\mathbf{C}$ which has received much popular as well as mathematical interest.

It is conjectured that the interior of $M$ consists of hyperbolic components for which the corresponding polynomial $P_{c}(z)$ has an attractive periodic point $\xi(c)$ of fixed period. For example, we may compute the $c$ values for which $P_{c}(z)$ has an attractive fixed point by solving $P_{c}(z)=z$ with the restriction $\left|P_{c}^{\prime}(z)\right|<1$. The solution consists of the open cardioid with boundary given by $\frac{1}{2} e^{i t}-\frac{1}{4} e^{2 i t}$ for $0 \leq t<2 \pi$. This cardioid is a hyperbolic component of $M$ which consists of those values of $c$ for which $P_{c}^{\circ n}(0)$ converges to an attractive fixed point as $n \rightarrow \infty$.

If $W$ is a hyperbolic component of period $n$, then there is an analytic bijection called the Hubbard-Douady map of $W$ onto the unit disk $D$

$$
\rho: W \rightarrow D
$$

given by

$$
c \mapsto \frac{d}{d z} P_{c}^{\circ n}(z)
$$

evaluated at $z=\xi(c)$. Moreover, the Hubbard-Douady map $\rho$ extends continuously to a homeomorphism

$$
\bar{\rho}: \bar{W} \rightarrow \bar{D}
$$

(see [1]). An explicit formula for $\bar{\rho}^{-1}$ can easily be computed for hyperbolic components of periods 1 and 2. We will find an explicit formula for $\bar{\rho}^{-1}$ in the period 3 case and discuss the period 4 case.

\section{THE PERIODIC POINTS}

If $\xi$ is a periodic point of $P_{c}(z)$ of period $n$, then it is a root of the degree $2^{n}$ polynomial

$$
P_{c}^{\circ n}(z)-z=0 .
$$

However, the roots of (1) will also contain periodic points of any period $d$ which divides $n$. This means that if $d \mid n$, then $\left(P_{c}^{\circ d}(z)-z\right) \mid\left(P_{c}^{\circ n}(z)-z\right)$, and therefore

$$
\frac{P_{c}^{o n}(z)-z}{P_{c}^{o d}(z)-z}
$$

is a polynomial.

We let $F_{n}(z)$ denote the polynomial whose roots are the periodic points of $P_{c}$ with minimal period $n$. We think of $F_{n}(z)$ as a polynomial of the field $\mathbf{Q}(c)$.

Remarks. 1. If $n$ is prime, then

$$
F_{n}(z)=\frac{P_{c}^{\circ n}(z)-z}{P_{c}(z)-z} .
$$

2. In general

$$
P_{c}^{\circ n}(z)-z=\prod_{d \mid n} F_{d}(z)
$$


so

$$
2^{n}=\sum_{d \mid n} \operatorname{deg} F_{d} .
$$

3. Using the Möbius inversion formula gives

$$
\operatorname{deg} F_{n}=\sum_{d \mid n} \mu\left(\frac{n}{d}\right) 2^{d}
$$

where $\mu$ is the Möbius function.

We can compute

$$
\begin{aligned}
F_{3}(z)= & z^{6}+z^{5}+(1+3 c) z^{4}+(1+2 c) z^{3}+\left(3 c^{2}+3 c+1\right) z^{2} \\
& +\left(c^{2}+2 c+1\right) z+c^{3}+2 c^{2}+c+1
\end{aligned}
$$

2.1. The multiplier equations. We are seeking a relationship between the parameter $c$ and the multiplier $\rho=\frac{d}{d z} P_{c}^{\circ n}(z)$ evaluated at the periodic points. If $\xi$ is a solution of equation (1), then since $P_{c}^{\prime}(z)=2 z$, the chain rule gives

$$
\rho=2^{n} \prod_{i=0}^{n-1} P_{c}^{\circ i}(\xi)
$$

The hyperbolic components are thus exactly the $c$ values for which $|\rho|<1$. We will work with the quantity $\lambda=2^{-n} \rho$ which makes the computations cleaner.

In principle, it is easy to find the relationship between $c$ and $\lambda$ by eliminating the indeterminate $z$ using the resultant of the polynomials

$$
\lambda-\prod_{i=0}^{n-1} P_{c}^{\circ i}(z)
$$

and $F_{n}(z)$. For $n=1,2$, and 3, we get

$$
\begin{gathered}
E_{1}(\lambda, c)=c-\lambda(1-\lambda)=c-\lambda+\lambda^{2}, \\
E_{2}(\lambda, c)=-c+\lambda-1,
\end{gathered}
$$

and

$$
E_{3}(\lambda, c)=c^{3}+2 c^{2}+(1-\lambda) c+(1-\lambda)^{2} .
$$

The polynomials $E_{n}$ give the locus of parameters $c$ and multipliers $\lambda$ such that $P_{c}$ has an $n$-cycle with multiplier $\lambda$. At this point, we can, in principle, solve for $\lambda$ in terms of $c$ and be done. However, this leads to branching problems. We seek a branch-free, closed form solution for $c(\lambda)$, and for this, we must factor $F_{3}(z)$ explicitly. In fact, the resultants become large fast, and so in general, the relationship between $c$ and $\lambda$ must be computed by factoring the $F_{n}(z)$ explicitly (see the last section for $E_{4}$ ). Several people ([3], [5]) have carried out this computation up to $E_{7}$. We will outline this process in the period 3 case and use it to find the map $\bar{\rho}^{-1}: \bar{D} \rightarrow \bar{W}$.

2.2. Factoring periodic-point polynomials. The roots of $F_{n}(z)$ come in groups of periodic cycles:

$$
\begin{aligned}
& \xi, P_{c}(\xi), \ldots, P_{c}^{\circ n-1}(\xi) \\
& \eta, P_{c}(\eta), \ldots, P_{c}^{\circ n-1}(\eta) \\
& \theta, P_{c}(\theta), \ldots, P_{c}^{\circ n-1}(\theta)
\end{aligned}
$$


so that

$$
F_{n}(z)=\prod_{i}\left(z-P_{c}^{\circ i}(\xi)\right) \prod_{i}\left(z-P_{c}^{\circ i}(\eta)\right) \prod_{i}\left(z-P_{c}^{\circ i}(\theta)\right) \cdots
$$

Let $\alpha, \beta, \gamma, \ldots$ denote a generic $n$-cycle $\xi, P_{c}(\xi), \ldots, P_{c}^{\circ n-1}(\xi)$, and define the associated cycle polynomial $T(z)$ by

$$
T(z)=\prod\left(z-P_{c}^{\circ i}(\xi)\right)=z^{n}-\sum \alpha z^{n-1}+\sum \alpha \beta z^{n-2}-\sum \alpha \beta \gamma z^{n-3}+\cdots,
$$

where $\sum \alpha, \sum \alpha \beta, \ldots$ denote the elementary symmetric functions associated with the periodic cycle. To each cycle, we associate a parameter $\Omega_{\xi}=\sum \alpha=$ $\sum P_{c}^{\circ i}(\xi)$ which indexes the cycle polynomial

$$
F_{n}(z)=\prod_{\Omega} T_{\Omega}(z),
$$

where the product is taken of all the roots of a resolvent equation $\operatorname{Res}(z)=$ $\prod_{\xi \in \Xi}\left(z-\Omega_{\xi}\right)$, and $\Xi$ is a set containing one element of each periodic cycle. The next proposition shows that all the coefficients of $T_{\Omega}$ can be written in terms of $\Omega$.

Proposition 1 (Lagrange). $T_{\Omega_{\xi}}(z) \in \mathbf{Q}(c)\left(\Omega_{\xi}\right)[z]$ for all but finitely many values of $c$.

Proposition 2. $\operatorname{Res}(z) \in \mathbf{Q}(c)[z]$.

Proof. Let $E$ be the splitting field of $F_{n}(z)$ over $\mathbf{Q}(c)$. This is a Galois extension of $\mathbf{Q}(c)$ and of $\mathbf{Q}(c)\left(\Omega_{\xi}\right)$. Choose $\sigma \in \operatorname{Gal}\left(E, \mathbf{Q}(c)\left(\Omega_{\xi}\right)\right)$ and $c \in \mathbf{C}-\Delta$, where $\Delta$ is the (finite) zero set of the discriminant of $F_{n}$. Now, $\sigma$ fixes $\Omega_{\xi}$, so

$$
\sigma(\xi)=P_{c}^{\circ i}(\xi)
$$

for some $i$. Thus, if $f$ is any symmetric function of the points in a periodic cycle, $\sigma(f)=f$. But the coefficients of $T_{\Omega_{\xi}}$ are precisely these symmetric functions.

The proof of Proposition 2 is the same. In this case, we consider the action of $\operatorname{Gal}(E, \mathbf{Q}(c))$ on the coefficients of $\operatorname{Res}(z)$.

We are interested in the symmetric function which is the product

$$
\alpha \beta \gamma \ldots=\prod_{i=0}^{n-1} P_{c}^{\circ i}(\xi),
$$

since this is exactly $\lambda$.

We now compute a key relationship between the symmetric functions $\sum \alpha$, $\sum \alpha \beta$ of the periodic cycle

$$
\Omega=\sum \alpha=\sum P_{c}(\alpha)=\sum\left(\alpha^{2}+c\right),
$$

so

$$
\sum \alpha^{2}=\Omega-n c
$$

But

$$
\Omega^{2}=\left(\sum \alpha\right)^{2}=\sum \alpha^{2}+2 \sum \alpha \beta
$$


so

$$
\sum \alpha \beta=\frac{1}{2}\left(\Omega^{2}-\Omega+n c\right) .
$$

By working with $F_{3}(z)$, lengthy direct calculations show that (for $n=3$ )

$$
\sum \alpha \beta=c-\Omega-1
$$

and

$$
\sum \alpha \beta \gamma=\alpha \beta \gamma=\lambda=c \Omega+c+1 \text {. }
$$

In this case, we obtain a factorization of $F_{3}(z)$ into two cubics in $\mathbf{Z}(c)(\Omega)[z]$. Namely,

$$
F_{3}(z)=\prod_{\{\Omega \mid \operatorname{Res}(\Omega)=0\}} z^{3}-\Omega z^{2}-(\Omega-c+1) z-c \Omega-c-1,
$$

where $\operatorname{Res}(\Omega)=\Omega^{2}+c+2$ follows from equations (2) and (3). The roots of each cubic are precisely the points of one periodic cycle.

Remark. In principle, this factorization method is due to Lagrange. In fact, the period 3 (and period 4) case appeared over 100 years ago in books by E. Netto (see, for example, [4]).

The relations (2), (3), and (4) between the symmetric functions also lead to

$$
c=-2-\Omega-\Omega^{2}
$$

and

$$
\lambda=-1-3 \Omega-2 \Omega^{2}-\Omega^{3} .
$$

We now take a brief look at the solutions of cubics.

\section{The CUBIC SOLUTION}

The reader may verify that the solution of

$$
z^{3}+a z+b=0
$$

is given by

$$
z=2 \sqrt{\frac{a}{3}} \sinh \left(\frac{1}{3} \operatorname{Arcsinh}\left(-\frac{b}{2 \gamma}\right)+\frac{2 k \pi i}{3}\right), \quad k=0,1,2,
$$

where $\gamma^{2}=\frac{a^{3}}{27}$. This may be done by letting $z=2 r \sinh \theta$ and using the identity

$$
\sinh (3 \theta)=3 \sinh \theta+4 \sinh ^{3} \theta
$$

\section{THE PERIOD 3 COMPONENTS}

The curve

$$
E_{3}(\lambda, c)=c^{3}+2 c^{2}+(1-\lambda) c+(1-\lambda)^{2}=0
$$

is unicursal with a unique double point at $c=0, \lambda=1$. The parameterization given by equations (5) and (6) gives a closed form solution for $\bar{\rho}^{-1}$ which is 
free from branching. We can use the solution in Section 3 to solve equation (6), giving

$$
\Omega=-\frac{2}{3}+\frac{2 \sqrt{5}}{3} \sinh \left(\omega(\rho)+\frac{2 k \pi i}{3}\right), \quad k=0,1,2,
$$

where

$$
\omega(\rho)=\frac{1}{3} \operatorname{Arcsinh}\left(\frac{88-27 \rho}{80 \sqrt{5}}\right) .
$$

This, using equation (5), gives

$$
c_{k}(\rho)=-\frac{7}{4}-\frac{20}{9}\left[\sinh \left(\omega(\rho)+\frac{2 k \pi i}{3}\right)-\frac{1}{4 \sqrt{5}}\right]^{2}, \quad k=0,1,2 .
$$

Figure 1 shows two of the period 3 component boundaries, plotted as the image of the unit circle by the solutions above. Figure 2 shows the hyperbolic components of periods 1,2 , and 3 derived from $E_{1}, E_{2}$, and the solutions above.
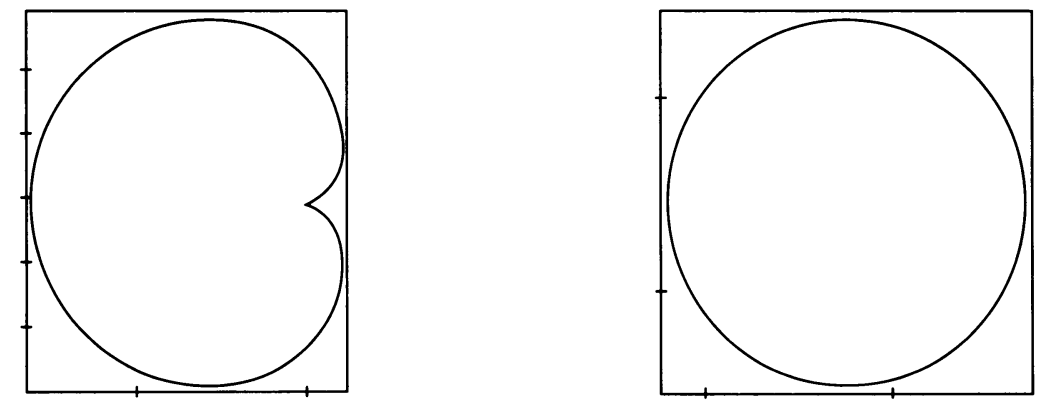

FIGURE 1. The boundary of two of the period 3 components of the Mandelbrot set

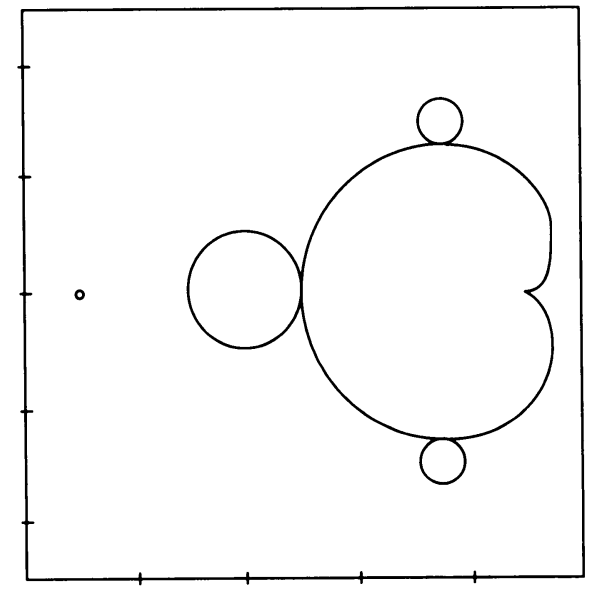

FIGURE 2. The boundary of the hyperbolic components of period 1, 2, and 3 of the Mandelbrot set 


\section{THE PERIOD 4 CASE}

Using the invariance of the symmetric functions $\sum \alpha \beta \gamma \ldots$ under applications of $P_{c}$, it is possible to factor

$$
\begin{aligned}
F_{4}(z)= & z^{12}+6 c z^{10}+z^{9}+\left(15 c^{2}+3 c\right) z^{8}+4 c z^{7}+\left(12 c^{2}+20 c^{3}+1\right) z^{6} \\
& +\left(6 c^{2}+2 c\right) z^{5}+\left(15 c^{4}+18 c^{3}+3 c^{2}+4 c\right) z^{4}+\left(4 c^{2}+4 c^{3}+1\right) z^{3} \\
& +\left(5 c^{2}+6 c^{3}+6 c^{5}+12 c^{4}+c\right) z^{2}+\left(c^{4}+2 c^{3}+c^{2}+2 c\right) z \\
& +3 c^{5}+c^{6}+3 c^{3}+3 c^{4}+2 c^{2}+1 \\
= & \prod_{\Omega} z^{4}-\Omega z^{3}+\frac{1}{2}\left(\Omega^{2}-\Omega+4 c\right) z^{2}+\frac{1}{2}\left(\Omega^{2}+(1-2 c) \Omega+2\right) z \\
& +\frac{1}{2} c \Omega^{2}+\frac{1}{2} c \Omega+c^{2}+c+1,
\end{aligned}
$$

where $\operatorname{Res}(z)=z^{3}+(4 c+3) z+4$.

It is then possible to compute

$$
E_{4}(\lambda, c)=c^{6}+3 c^{5}+(3+\lambda) c^{4}+(3+\lambda) c^{3}+\left(2-\lambda-\lambda^{2}\right) c^{2}+(1-\lambda)^{3},
$$

which admits the rational parameterization

$$
\begin{gathered}
c=-\frac{3}{4}-\Omega^{-1}-\frac{1}{4} \Omega^{2}, \\
\lambda=\frac{16+8 \Omega+5 \Omega^{2}-6 \Omega^{3}-4 \Omega^{4}-2 \Omega^{5}-\Omega^{6}}{16 \Omega^{2}} .
\end{gathered}
$$

We would like to solve equation (9) for $\Omega$ in terms of $\lambda$ and substitute this in equation (8). However, this will be difficult because, in general, the polynomial

$$
\Omega^{6}+2 \Omega^{5}+4 \Omega^{4}+6 \Omega^{3}+(16 \lambda-5) \Omega^{2}-8 \Omega-16,
$$

which follows from equation (9), has the symmetric group $S_{6}$ as its Galois group over $\mathbf{Q}(\lambda)$.

\section{REFERENCES}

1. A. Douady and J. H. Hubbard, Itération des polynômes quadratiques complexes, C. R. Acad. Sci. Paris 294 (1982).

2. P. Fatou, Sur les équations fonctionelles, Bull. Soc. Math. France 47 (1919); 48 (1920).

3. D. Giarrusso, unpublished notes, Cornell University, Ithaca, NY, 1989.

4. E. Netto, Theory of substitutions, Chelsea, New York, 1892.

5. J. Stephenson and D. T. Ridgway, Formulae for cycles in the Mandelbrot set II, Phys. A 190 (1992), 104-116.

Department of Mathematics, St. Lawrence University, Canton, New York 13617

E-mail address: dgiaeslumus.bitnet

Institute for Nonlinear Science, University of California, Sand Diego, la Jolla, CALIFORNIA 92093-0402

E-mail address: yfisher@uscd.edu 